\title{
Algoritmos para o Problema de Equilíbrio de Nash
}

\author{
Euda Mara da Silva Ferreria \\ Universidade Federal do Paraná, UFPR, \\ Curitiba, PR \\ E-mail: diretoria@facel.com.br \\ Luiz Carlos Matioli \\ Universidade Federal do Paraná - Departamento de Matemática \\ Curitiba,PR \\ E-mail: matioli@ufpr.br
}

17 de setembro de 2014

Resumo: Este artigo tem como objetivo apresentar algoritmos para solução de problemas de Equilíbrio de Nash (NEP). Para isto, são propostos dois novos algoritmos baseados na resolução de um sistema não linear $G(x)=0$, com $G: \mathbb{R}^{n} \rightarrow \mathbb{R}^{n}$ contínua mas não diferenciável em todos os pontos do dominio, sendo o primeiro algoritmo baseado, no Método de Newton, e o segundo em métodos Subgradientes. A origem desses algoritmos é o artigo [2], em que foi introduzido o método de Lagrangeano Aumentado, para a resolução de um Problema de Equilíbrio geral, em que o Problema Equilíbrio de Nash é um caso particular.

Palavras-chave: Problema de Equilíbrio de Nash; Métodos Subgradientes; Método de Newton; Problema de Equilíbrio; Algoritmos para solução de um NEP.

\section{Introdução}

O Problema de Equilíbrio de Nash (NEP) é um estudo interdisciplinar. Sua aplicação pode ser encontrada nas áreas de economia, matemática, engenharia, ciências da computação e pesquisa operacional. Devido à sua origem matemático-econômico, há várias aplicações no meio econômico e na Teoria dos Jogos. Poderá ser observado que existem mais pesquisa sobre a existência de soluções, do que boas implementações. Com o objetivo de avançar a pesquisa, são propostos dois novos algoritmos para resolver o NEP, cuja origem é o artigo de [2], em que foi introduzido o método de Lagrangeano Aumentado, para a resolução de um Problema de Equilíbrio geral.

\section{Métodologia para a Resolução do NEP}

O Problema de Equilíbrio de Nash consiste em $N$ jogadores, cada um é representado por $j$ e controla a variável $x^{j} \in \mathbb{R}^{n_{j}}$. As variáveis de decisão dos outros jogadores são representadas por $x^{-j}$. Portanto, o NEP consiste em resolver o problema (1) dado por:

$$
\min _{x^{j}} \quad \theta_{j}\left(x^{j}, x^{-j}\right) ; \quad \text { s.a } \quad x^{j} \in X_{j}\left(x^{-j}\right),
$$

onde $X_{j}\left(x^{-j}\right) \subseteq \mathbb{R}^{n_{j}}$ representa as jogadas estratégicas do jogador $j$. Sendo o conjunto $X_{j}\left(x^{-j}\right)$ dado por $X_{j}\left(x^{-j}\right)=\left\{x^{j} \in R^{n_{j}}: g\left(x^{j}, x^{-j}\right) \leq 0\right\}$, em que $g\left(., x^{-j}\right): \mathbb{R}^{n_{j}} \rightarrow \mathbb{R}^{m_{j}}$ representa o conjunto das restrições de todos os jogadores.

Em [2] foi introduzido métodos de Lagrangeano Aumentado nas versões Exata e Inexata para o Problema de Equilíbrio geral e como caso especial o NEP. A análise de convergência que os 
autores apresentam neste artigo está fortemente ligada aos métodos de ponto proximal para o Problema de Equilíbrio. A metodologia porposta para resolução do NEP consiste em dois novos algoritmos, baseados na resolução de um sistema não linear $G(x)=0$, sendo $G: \mathbb{R}^{n} \rightarrow \mathbb{R}^{n}$ contínua mas não diferenciável em todos os pontos do domínio.

O subproblema a ser resolvido, no algoritmo LIALEM apresentado em [2] para o caso diferenciável, é equivalente a resolução do sistema $G(x)=0$, dado por:

$$
G(x)=\gamma_{k}\left[x-x^{k}\right]+\nabla F_{x}(x)+\sum_{i=1}^{m} \max \left\{0, \lambda_{i}^{k}+\frac{h_{i}(x)}{\gamma_{k}}\right\} \nabla h_{i}(x)
$$

em que, $x^{k} \in \mathbb{R}^{n},\left\{\gamma_{k}\right\} \subset \mathbb{R}_{++}$é uma sequência limitada de parâmetros de regularização, as funções $h_{i}(x)$, diferenciáveis, em que $i=1, \ldots, m$ são as restrições do Problema de Equilíbrio, $\lambda_{i}^{k}, i=1, \ldots, m$ são os múltiplicadores de Lagrange e $\nabla F_{x}(x)$ obtido ao substituir $y$ por $x$ em $\nabla F_{x}(y)=\nabla_{y} f(x, y)$, sendo $f(x, y)$ a bi-função dada pelo Problema de Equilíbrio, diferenciável na variável $y$ para cada $x \in \mathbb{R}^{n}$ fixo.

\subsection{Aproximação Quadrática do Termo não Diferenciável}

A suavização da função $G(x)$ é realizada por meio de uma aproximação quadrática dos termos não diferenciáveis desta. Note que as componentes do sistema (2) podem ser reescritas na forma:

$$
G_{i}(x)=\left\{\begin{array}{lc}
\gamma_{k}\left[x_{i}-x_{i}^{k}\right]+\left(\nabla F_{x}(x)\right)_{i}+\sum_{i=1}^{m}\left(\lambda_{i}^{k}+\frac{h_{i}(x)}{\gamma_{k}}\right)\left(\nabla h_{i}(x)\right)_{i} & \text { se } \lambda_{i}^{k}+\frac{h_{i}(x)}{\gamma_{k}}>0 \\
\gamma_{k}\left[x_{i}-x_{i}^{k}\right]+\left(\nabla F_{x}(x)\right)_{i} & \text { caso contrário. }
\end{array}\right.
$$

para $i=1, \ldots, m,\left(\nabla F_{x}(x)\right)_{i}$ é a componente $i$ de $\nabla F_{x}(x)$ e $\left(\nabla h_{i}(x)\right)_{i}$ é a componente $i$ de $\nabla h_{i}(x)$. A função $G(x)$ só não é diferenciável para os índices $i$ em que $\lambda_{i}^{k}+\frac{h_{i}(x)}{\gamma_{k}}=0$.

Desta forma, o termo que envolve o máximo no somatório da $G(x)$ em (2) é o que deve ser suavizado. Será realizada uma suavização no intervalo $[-\eta, \eta]$, com $\eta>0$ e pequeno. Para tal, será considerada uma função quadrática $q(y)=a y^{2}+b y+c, \operatorname{com} a, b$ e $c$ a serem determinados de modo que a função ajustada seja continuamente diferenciável em todo $\mathbb{R}$. Considere a função $p_{i}: \mathbb{R} \rightarrow \mathbb{R}$ como:

$$
p_{i}\left(h_{i}(x)\right)=\max \left\{0, \lambda_{i}^{k}+\frac{h_{i}(x)}{\gamma_{k}}\right\}= \begin{cases}0, & \text { se } \lambda_{i}^{k}+\frac{h_{i}(x)}{\gamma_{k}} \leq 0 \\ \lambda_{i}^{k}+\frac{h_{i}(x)}{\gamma_{k}}, & \text { se } \lambda_{i}^{k}+\frac{h_{i}(x)}{\gamma_{k}}>0\end{cases}
$$

para cada $i=1, \ldots, m$, a aproximação quadrática para $p_{i}$ é dada por:

$$
\bar{p}_{i}\left(h_{i}(x)\right)= \begin{cases}0, & \text { se } \quad \lambda_{i}^{k}+\frac{h_{i}(x)}{\gamma_{k}} \leq-\eta \\ \frac{1}{4 \eta}\left(\lambda_{i}^{k}+\frac{h_{i}(x)}{\gamma_{k}}\right)^{2}+\frac{1}{2}\left(\lambda_{i}^{k}+\frac{h_{i}(x)}{\gamma_{k}}\right)+\frac{\eta}{4}, & \text { se } \quad-\eta<\lambda_{i}^{k}+\frac{h_{i}(x)}{\gamma_{k}} \leq \eta \\ \lambda_{i}^{k}+\frac{h_{i}(x)}{\gamma_{k}}, & \text { se } \quad \lambda_{i}^{k}+\frac{h_{i}(x)}{\gamma_{k}}>\eta,\end{cases}
$$

$\mathrm{e}$

$$
\bar{p}_{i}^{\prime}\left(h_{i}(x)\right)= \begin{cases}0, & \text { se } \lambda_{i}^{k}+\frac{h_{i}(x)}{\gamma_{k}} \leq-\eta \\ \frac{1}{2 \eta}\left(\lambda_{i}^{k}+\frac{h_{i}(x)}{\gamma_{k}}\right) \frac{\nabla h_{i}(x)}{\gamma_{k}}+\frac{\nabla h_{i}(x)}{2 \gamma_{k}}, & \text { se } \quad-\eta<\lambda_{i}^{k}+\frac{h_{i}(x)}{\gamma_{k}} \leq \eta \\ \frac{\nabla h_{i}(x)}{\gamma_{k}}, & \text { se } \quad \lambda_{i}^{k}+\frac{h_{i}(x)}{\gamma_{k}}>\eta .\end{cases}
$$

Finalmente, tem-se a função $G(x)$ suavizada:

$$
G(x)=\gamma_{k}\left[x-x^{k}\right]+\nabla F_{x}(x)+\sum_{i=1}^{m} \bar{p}_{i}\left(h_{i}(x)\right) \nabla h_{i}(x) .
$$

com $\bar{p}_{i}\left(h_{i}(x)\right)$ dada por (5). Desta forma, $G(x)$ dada por (7) se torna diferenciável. 
Proposição 2.1. Se $\eta \rightarrow 0$ então $\bar{p}_{i}\left(h_{i}(x)\right)$ dada em (5) coincide com $p_{i}\left(h_{i}(x)\right)$ dada em (4), para todo $i=1, \ldots, m$.

\subsection{Método de Newton para Resolução do Subproblema Gerado pelo Algo- ritmo de Equilíbrio}

O método de Newton será utilizado para resolver o subproblema gerado pelo algoritmo LIALEM apresntado em [2]. Ou seja, equivale a resolver sistema não linear $G(x)=0$, com $G(x)$ dada em (2). Desta forma, se $\eta$ for escolhido pequeno em (5) pela proposição 2.1, ter-se-á mantido os resultados já provados para o Problema de Equilíbrio. Na próxima proposição, será provado que escolhendo adequadamente o parâmetro de regularização a Jacobianda de $G(x)$, dada em (2), será positiva definida. Para isso, e para os próximos algoritmos a serem apresentados, será necessária a seguinte hipótese:

Hipótese 2.2. As funçôes $F_{x}: \mathbb{R}^{n} \rightarrow \mathbb{R}$, dada por $F_{x}=f(x, y)$ e $h_{i}: \mathbb{R}^{m} \rightarrow \mathbb{R}$, para $i=1, \ldots, m$, são diferenciáveis até segunda ordem.

Proposição 2.3. Considere a Hipótese 2.2 satisfeita e $G(x)$ dada em (5) e (7). Então, a Jacobiana de $G(x)$ é dada por:

$$
J G(x)=\gamma_{k} I_{n \times n}+\nabla^{2} F_{x}(x)+\sum_{i=1}^{m} \bar{p}_{i}^{\prime}\left(h_{i}(x)\right) \nabla h_{i}(x)+\sum_{i=1}^{m} \bar{p}_{i}\left(h_{i}(x)\right) \nabla^{2} h_{i}(x) .
$$

em que $\bar{p}_{i}^{\prime}\left(h_{i}(x)\right)$, dada em (6), é a derivada de $\bar{p}_{i}\left(h_{i}(x)\right)$ e $I_{n \times n}$ é a matriz identidade. Além disso, para $i \in\{1,2, \ldots, m\}$ tem se:

(a) Se $\lambda_{i}^{k}+\frac{h_{i}(x)}{\gamma_{k}} \leq-\eta$ ou $\lambda_{i}^{k}+\frac{h_{i}(x)}{\gamma_{k}}>\eta$ então $J G(x)>0$, para todo $x \in \mathbb{R}^{n}$.

(b) $S e-\eta<\lambda_{i}^{k}+\frac{h_{i}(x)}{\gamma_{k}} \leq \eta$ e $x \in \mathbb{R}^{n}$ estiver fixado, então existe $\bar{k}$ tal que para todo $k>\bar{k}$ $J G(x)>0$.

Algoritmo 2.4. PROBLEMA DE EQUILIBRIO COMBINADO COM NEWTON PARA RESOLVER O SUBPROBLEMA

Dados $x^{0} \in \mathbb{R}^{n}, \lambda^{0}>0, \eta>0, \varepsilon_{1}>0, \varepsilon_{2}>0$ e $\gamma^{0}>0$

$k=0$ e aux $=1$,

Enquanto aux $=1$

Faça $\ell=0$, e $z^{\ell}=x^{k}$,

Avalie $G\left(z^{\ell}\right)$, dada em (5) e (7)

Enquanto $\left\|G\left(z^{\ell}\right)\right\|>\varepsilon_{1}$

Avalie $J G\left(z^{\ell}\right) \quad\left(J G\left(z^{\ell}\right)\right.$ é a Jacobiana da $G(x)$ em $\left.z^{\ell}\right)$

Determine $d^{\ell}=-\left(J G\left(z^{\ell}\right)\right)^{-1} G\left(z^{\ell}\right), \quad$ (direção de Newton)

Faça $z^{\ell+1}=z^{\ell}+d^{\ell}$ $\ell=\ell+1$, e Avalie $G\left(z^{\ell}\right)$, dada em (5) e (7)

Fim enquanto

Faça $x^{k+1}=z^{\ell+1}, e$

$$
\lambda_{i}^{k+1}=\max \left\{0, \lambda_{i}^{k}+\frac{h\left(x^{k+1}\right)}{\gamma^{k}}\right\}, \quad i=1, \ldots, m,
$$

Se $\left\|\left(x^{k+1}, \lambda^{k+1}\right)-\left(x^{k}, \lambda^{k}\right)\right\| \leq \varepsilon_{2}$

aux $=0$,

Fim se

Faça $k=k+1$,

Fim enquanto

Para os algoritmos 2.4 e 2.6 (a ser apresentado na sequência), serão considerados os critérios de parada $\varepsilon_{1}>0$ e $\varepsilon_{2}>0$ respctivamente para o subproblema interno e externo. Para o subproblema interno $\ell$ é o número de iterações que se utiliza para determinar a solução de 
$G(x)=0$ com precisão $\varepsilon_{1}$. Por outro lado, $k$ é o número de iterações para o problema externo com precisão $\varepsilon_{2}$. Desta forma, o total de iterações que o algoritmo utiliza para resolver o problema é $k+\ell . \quad \lambda \geq 0$ é o multiplicador de Lagrange associado às restrições de igualdade $\mathrm{e}$ $\gamma>0$ é o parâmetro de regularização (no dual) ou o parâmetro de penalidade (no primal). Sobre a atualização do parâmetro $\gamma>0$, é feita uma descrição na seção de implementação numérica.

O teorema 2.5 prova que a direção de Newton, gerada pelo algoritmo 2.4, está bem definida.

Teorema 2.5. Considere a Hipótese 1 satisfeita e as funções $F_{x}$ e $h_{i}(x)$, para $i=1, \ldots, m$, definidas no Problema de Equíbrio, convexas. Então, a cada iteração $\ell$ do algoritmo 7 a direção de Newton $d^{\ell}=-\left(J G\left(z^{\ell}\right)\right)^{-1} G\left(z^{\ell}\right)$ está bem definida.

\subsection{Método Subgradiente para Resolução do Subproblema Gerado pelo Al- goritmo de Equilíbrio}

A metodologia consiste no uso de métodos de Subgradientes para resolver o sistema não linear e não diferenciável $G(x)=0$ sendo $G(x)$ dada em (2). Para usar os métodos Subgradientes este sistema será transformado em um problema de minimização da forma:

$$
\min \left\{f(x): x \in \mathbb{R}^{n}\right\},
$$

sendo $f: \mathbb{R}^{n} \rightarrow \mathbb{R}$ dada por $f(x)=\frac{1}{2}\|G(x)\|^{2}$, em que $G: \mathbb{R}^{n} \rightarrow \mathbb{R}^{n}$ é dada por (2). Considere $g: \mathbb{R}^{n} \rightarrow \mathbb{R}$ a função definida por $g(x)=\frac{1}{2}\|x\|^{2}$. Assim, a função $f(x)$ pode ser reescrita como a função composta $f(x)=g(G(x))$. Como pode ser observado $g(x)$, é diferenciável e estritamente convexa. Mas o mesmo não acontece, em geral, com a função $f(x)=g(G(x))$, pois $G(x)$ pode ser não convexa e é não diferenciável em pontos do seu domínio. Desta forma, não é possível aplicar diretamente os métodos Subgradientes. Para isso, será suavizado o termo não diferenciável, conforme a seção, Aproximação Quadrática, para que $G(x)$ dada em (2) se torne diferenciável e, então, imitar o método do Gradiente para calcular um Subgradiente. Neste caso, em qualquer ponto $x \in \mathbb{R}^{n}$ ter-se-á:

$$
\partial f(x)=\nabla f(x)=\nabla G(x)^{T} G(x) .
$$

Um passo do método Subgradiente para resolver $G(x)$, parte de um ponto já conhecido $x^{k} \in \mathbb{R}^{n}$ e determina o próximo ponto utilizando $x^{k+1}=x^{k}-\alpha_{k} \partial f\left(x^{k}\right)$, sendo $\alpha_{k}$ o tamanho do passo e $\partial f\left(x^{k}\right)$ o Subgradiente no ponto $x^{k}$. A seguir é apresentado o algoritmo para resolver o NEP com os métodos de Subgradientes, para resolver o subproblema $G(x)=0$ dada em (7). No algoritmo 2.6, será apresentado o algoritmo básico, no sentido que utiliza procedimentos simples para atualizar o tamanho do passo (ver [6]).

Algoritmo 2.6. PROBLEMA DE EQUILÍBRIO COMBINADO COM O MÉTODO SUBGRADIENTE PARA RESOLVER O SUBPROBLEMA

Dados $x^{0} \in \mathbb{R}^{n}, \lambda^{0}>0, \alpha>0, a>0$ (coeficiente de dilatação), $\varepsilon_{1}>0, \varepsilon_{2}>0$ e $\gamma^{0}>0$

$k=0$ e aux=1,

Enquanto aux $=1$

Faça: $\ell=0$, e $z^{\ell}=x^{k}$,

Avalie $G\left(z^{\ell}\right)$, dada em (5) e (7)

Enquanto $\left\|G\left(z^{\ell}\right)\right\|>\varepsilon_{1}$

Avalie $J G\left(z^{\ell}\right) \quad\left(J G\left(z^{\ell}\right)\right.$ é a Jacobiana da $G(x)$ em $\left.z^{\ell}\right)$

Determine $\partial f\left(z^{\ell}\right)=-J G\left(z^{\ell}\right)^{T} G\left(z^{\ell}\right)$, (um subgradiente de $f(x)$

em $z^{\ell}$, com $f=g\left(G\left(z^{\ell}\right)\right)$

Escolher $\alpha_{\ell}$ apropriado

Faça: $d_{\ell}=\frac{\partial f\left(z^{\ell}\right)}{\left\|\partial f\left(z^{\ell}\right)\right\|}, \quad e$

$$
z^{\ell+1}=z^{\ell}+\alpha_{\ell} d_{\ell}
$$


Avalie $G\left(z^{\ell+1}\right)$, dada em (5) e (7)

Faça $\ell=\ell+1$,

Fim enquanto

Faça: $x^{k+1}=z^{\ell+1}, e$

$$
\lambda_{i}^{k+1}=\max \left\{0, \lambda_{i}^{k}+\frac{h\left(x^{k+1}\right)}{\gamma^{k}}\right\}, \quad i=1, \ldots, m,
$$

Se $\left\|\left(x^{k+1}, \lambda^{k+1}\right)-\left(x^{k}, \lambda^{k}\right)\right\| \leq \varepsilon_{2}$

aux $=0$,

Fim se

Faça $k=k+1$,

Fim enquanto

\section{Resultados Numéricos}

Os métodos Subgradientes utilizados para solução do subproblema, conforme o algoritmo 2.6, terão como escolha do tamanho do passo $\alpha_{k}$ e da direção $d^{k}$ os resultados apresentados por [6] e por [5]. Nas implementações foram considerados $\gamma^{k}=\frac{1}{250}$ para o algoritmo do Problema de Equilíbrio combinado com os métodos de Newton e Dilatação de Espaço. Para os demais métodos utilizou-se $\gamma^{k}=1$. O coeficiente de dilatação para o método de Dilatação de Espaço $a=0.1$ e para o método Elipsóides $a=1, \eta=0.1$ para todos os métodos implementados. O critério de parada para o subproblema interno $\left\|G\left(x^{k}\right)\right\|<\varepsilon_{1}$, o critério de parada para o Problema de Equilíbrio $\left\|\left(x^{k+1}, \lambda^{k+1}\right)-\left(x^{k}, \lambda^{k}\right)\right\| \leq \varepsilon_{2}$, onde $\varepsilon_{1}=\varepsilon_{2}=10^{-4}$ e o número máximo de iterações no laço interno que resolve $G(x)$ igual a 1000 .

Exemplo 3.1. POLUIÇÃO DE UMA BACIA HIDROGRÁFICA (River Basin Pollution)

Considere o problema de poluição de uma bacia hidrográfica apresentado em [3],[7] e [4], com três jogadores $j=1,2,3$. A resolução do problema consiste em minimizar a funçẫo objetivo dado por:

$$
\begin{gathered}
\theta_{j}(x)=c_{2 j} x_{j}^{2}+d_{2} x_{j}\left(x_{1}+x_{2}+x_{3}\right)-b_{j} x_{j}= \\
=c_{2 j} x_{j}^{2}+0,01 x_{j}\left(x_{1}+x_{2}+x_{3}\right)-b_{j} x_{j} \quad(j=1,2,3),
\end{gathered}
$$

com $c_{2 j}=(0.01,0.05,0.01), b_{j}=d_{1}-c_{1 j}=(2.90,2.88,2.85)$ e sujeita às restriçôes dadas por:

$$
q_{l}(x)=\left\{\begin{array}{c}
3,25 x_{1}+1,25 x_{2}+4,125 x_{3} \leq 100 \\
2,291 x_{1}+1,5625 x_{2}+2,8125 x_{3} \leq 100
\end{array}\right.
$$

Na implementação deste problema, foram considerados para todos os métodos $x^{0}=[5,9,3]^{T}$, $\lambda^{0}=\left[1, \frac{3}{2}\right]^{T}, \gamma^{0}=2$.

\begin{tabular}{|c|c|c|c|c|}
\hline Métodos & $x^{*}$ & $\|G(x)\|$ & Ite. Ext. $(\mathrm{k})$ & $k+\ell$ \\
\hline Subgradiente Básico & {$[21.1487 ; 16.0276 ; 2.7229]^{T}$} & $9.8729 \mathrm{e}-005$ & 155 & 66922 \\
\hline Ponto Ótimo & {$[21.1397 ; 16.0279 ; 2.7300]^{T}$} & $9.9665 \mathrm{e}-005$ & 274 & 73146 \\
\hline Método de Elipsóides & {$[21.1390 ; 16.0280 ; 2.7305]^{T}$} & $8.8532 \mathrm{e}-005$ & 267 & 39742 \\
\hline Dilatação do Espaço & {$[21.1428 ; 16.0279 ; 2.7276]^{T}$} & $8.4908 \mathrm{e}-005$ & 06 & 55 \\
\hline Newton & {$[21.1430 ; 16.0279 ; 2.7274]^{T}$} & $8.4132 \mathrm{e}-005$ & 06 & 19 \\
\hline
\end{tabular}

Tabela 1: Solução Ótima do Exemplo 3.1.

Exemplo 3.2. (ver [1], p.178)

Considere um jogo com dois jogadores, $N=2$ com $n_{1}=1$ e $n_{2}=1$. Assim, cada jogador controla uma variável; por simplicidade define-se $x_{1}^{1}=x^{1}$ e $x_{1}^{2}=x^{2}$. Assumindo que os problemas dos jogadores são: 


$$
\min _{x^{1}}\left(x^{1}-1\right)^{2} ; \quad \text { s.a } \quad x^{1}+x^{2} \leqslant 1 \quad \min _{x^{2}}\left(x^{2}-\frac{1}{2}\right)^{2} ; \quad \text { s.a } \quad x^{1}+x^{2} \leqslant 1 .
$$

Para o exemplo (3.2), foi considerado $x^{0}=[0,0]^{T}, \lambda^{0}=1, \gamma^{0}=2$.

\begin{tabular}{|c|c|c|c|c|}
\hline Métodos & $x^{*}$ & $\|G(x)\|$ & Iter. Ext. $(\mathrm{k})$ & $k+\ell$ \\
\hline Subgradiente Básico & {$[0.7500 ; 0.2500]^{T}$} & $1.1028 \mathrm{e}-005$ & 48 & 13402 \\
\hline Ponto Ótimo & {$[0.7500 ; 0.2500]^{T}$} & $5.8968 \mathrm{e}-005$ & 11 & 144 \\
\hline Elipsóides & {$[0.7500 ; 0.2500]^{T}$} & $7.0114 \mathrm{e}-005$ & 17 & 1116 \\
\hline Dilatação do Espaço & {$[0.7500 ; 0.2500]^{T}$} & $1.4356 \mathrm{e}-006$ & 4 & 17 \\
\hline Newton & {$[0.7500 ; 0.2500]^{T}$} & $1.1069 \mathrm{e}-014$ & 4 & 10 \\
\hline
\end{tabular}

Tabela 2: Solução Ótima do Exemplo 3.2.

\section{Conclusão}

Pelos testes realizados e os resultados mostrados, a metodologia proposta e os algoritmos implementados neste trabalho tiveram um excelente desempenho, em relação aos mesmos exemplos aplicados na literatura da área. Portanto, a metodologia proposta mostrou-se promissora, como pode ser visto nos testes numéricos apresentados em Resultados Numéricos e oferece dois novos algoritmos como opções a mais para os interessados na solução do Problema de Equilíbrio de Nash (NEP).

\section{Referências}

[1] FACCHINEI, F.; KANZOW, C. Generalizes Nash Equilibrium Problems. Preprint 290. Ann Oper Res 175. p. 177211, 2010.

[2] IUSEM, A. N.; NASRI, M. Augmented Lagarangian methods for equilibrium problems. nov. 2007a.

[3] KRAWCZYK,J.B.; URYASEV, S. Relaxation algorithms to find Nash equilibria whit economic applications.Baltzer Science Publishers BV.Environmental Modeling and Assessment 5(2000). p:63-73. Setembro, 1999.

[4] MATIOLI, C. L.; SOSA, W.; YUAN, J. A numerical algorithm for solutions of a Generalized Nash Equilibrium Problem. Computational Otimization and Applications, p. 1-12, 2011.

[5] MIMOUX, M.; Mathematical Programming Theory and Algorithms, John Wiley and Sons, 1986.

[6] POLYAK, B. T.;Introduction to Optimization. Optimization Software, Inc. New York, 1987.

[7] SANTOS P.; SCHEIMBERG S. An inexact subgradient algorithm for Equilibrium Problems. Computational Applied Mathematics, Volume 30, N.1,p. 91-107. 2011.

[8] TRAN, D. Q. AND DUNG, M. L. AND NGUYEN, V. H. Extragradient algorithms extended to equilibrium problems.Bibliotheque Univ De Namur. março, 2006. 